\title{
Effect of ex vivo expanded recipient regulatory $T$ cells on hematopoietic chimerism and kidney allograft tolerance across MHC barriers in cynomolgus macaques
}

\author{
Raimon Duran-Struuck, DVM PhD" ${ }^{\# 1}$, Hugo P Sondermeijer, MD MS ${ }^{\# 1}$, Leo Bühler, MD4,

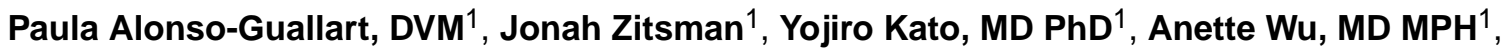 \\ Alicia N. McMurchy, PhD ${ }^{3}$, David Woodland, MD ${ }^{1}$, Adam Griesemer, MD ${ }^{1}$, Mercedes \\ Martinez, MD¹, Svetlan Boskovic, MD², Tatsuo Kawai, MD PhD², A. Benedict Cosimi, MD², \\ Cheng-Shie Wuu, PhD ${ }^{1}$, Andrea Slate, DVM ${ }^{1}$, Markus Mapara ${ }^{1}$, Sam Baker, DVM ${ }^{1}$, Rafal \\ Tokarz, PhD ${ }^{1}$, Vivette D'Agati, MD ${ }^{1}$, Scott Hammer, MD¹, Marcus Pereira, MD¹, W. lan \\ Lipkin, MD ${ }^{1}$, Thomas Wekerle, $\mathbf{M D}^{5}$, Megan Levings, $\mathrm{PhD}^{3}$, and Megan Sykes, $\mathbf{M D}^{1}$ \\ ${ }^{1}$ Columbia University Medical Center, New York, NY, United States ${ }^{2}$ Massachusetts General \\ Hospital, Boston. MA, United States ${ }^{3}$ University of British Columbia, Vancouver, British Columbia, \\ Canada ${ }^{4}$ Geneva University Hospital, Geneva, Switzerland ${ }^{5}$ Section of Transplantation \\ Immunology, Department of Surgery, Medical University of Vienna, Vienna, Austria. \\ \# These authors contributed equally to this work.
}

\section{Abstract}

Background-Infusion of recipient regulatory $\mathrm{T}$ cells (Treg) promotes durable mixed hematopoietic chimerism and allograft tolerance in mice receiving allogeneic BMT with minimal conditioning. We applied this strategy in a Cynomolgus macaque model.

\begin{abstract}
Methods-CD4+CD25high Treg that were polyclonally expanded in culture were highly suppressive in vitro and maintained high expression of FoxP3. Eight monkeys underwent nonmyeloablative conditioning and MHC-mismatched BMT with or without Treg infusion. Renal transplantation (from the same BMT donor) was performed 4 months post-BMT without immunosuppression to assess for robust donor-specific tolerance.
\end{abstract}

Results-Transient mixed chimerism, without significant $\mathrm{T}$ cell chimerism, was achieved in the animals that received BMT without Treg $(\mathrm{N}=3)$. In contrast, the 2 of 5 recipients of Treg + BMT that were evaluable displayed chimerism in all lineages, including $\mathrm{T}$ cells, for up to 335 days postBMT. Importantly, in the animal that survived long-term, $>90 \%$ of donor T cells were CD45RA +CD31+, suggesting they were new thymic emigrants. In this animal, the delayed (to 4 months)

Corresponding author: ms3976@ columbia.edu.

Disclosure: Authors do not report any conflict of interest pertaining to the work shown in this manuscript.

RDS, HS, MS performed research, analyzed data and wrote the manuscript. LB, PAG, JZ performed research, analyzed data. RT, WIL performed CMV testing. YK, BC, SV, TK, AG performed the surgeries and collected samples and participated in data analysis. CSW provided the irradiation. SH, MP participated in research and CMV assessment. ALM and ML provided Treg expertise and artificial APCs. AS, SB participated in NHP care. MM participated in research. MM participated in research as the BMT consultant. TW participated in research and manuscript. VA performed pathology. 
donor kidney graft was accepted $>294$ days without immunosuppression, whereas non-Treg BMT recipients rejected delayed donor kidneys within 3-4 weeks. Early cytomegalovirus reactivation and treatment was associated with early failure of chimerism, regardless of Treg administration.

Conclusions-Our studies provide proof-of-principle that, in the absence of early CMV reactivation (and BM-toxic antiviral therapy), co-transplantation of host Treg can promote prolonged and high levels of multilineage allogeneic chimerism and robust tolerance to the donor.

\section{INTRODUCTION}

CD4+FoxP3+ regulatory T cells (Treg) modulate auto- and alloimmune responses 1,2,3-5. Induction of kidney allograft tolerance, via transient mixed hematopoietic chimerism and nonmyeloablative conditioning has been achieved in large animal models ${ }^{6}$, and humans ${ }^{7}$. However, kidney allogaft tolerance was achieved in only 60-70\% of cynomolgus monkeys (cynos) and humans, and tolerance could not be readily extended to islet, heart or lung allografts in monkeys ${ }^{8-10}$. While durable mixed chimerism has been achieved with total lymphoid radiation, anti-thymocyte globulin and donor kidney transplantation in the HLAidentical transplant setting, this approach has not yet succeeded in achieving durable chimerism or tolerance across HLA barriers ${ }^{11-14}$. Another approach achieves renal allograft tolerance with development of full donor chimerism across extensive HLA barriers ${ }^{15,16}$, but the full donor chimerism likely reflects the more rigorous and potentially toxic host conditioning and/or graft-vs-host reactivity of the infused donor T cells, which eliminates recipient hematopoiesis, and high rates of opportunistic infection were observed ${ }^{17}$. Mixed chimerism, in contrast, provides a steady supply of recipient-derived antigen presenting cells (APCs), conferring superior ability to mount cytotoxic $\mathrm{T}$ cell responses that clear viral infections compared to full chimeras ${ }^{17-20}$. Thus, the reliable achievement of durable mixed chimerism across HLA barriers, with its potential to induce tolerance to any type of donor organ and to cure congenital hematologic disorders, remains an important and elusive goal in humans 21,22 .

In mice, adoptive transfer of recipient blood-derived natural Treg (referred as Treg) at the time of BMT with minimal conditioning regimen permitted the establishment of permanent hematopoietic mixed chimerism and skin allograft tolerance ${ }^{23-26}$. We have adapted the use of Treg for the above-mentioned cyno model that otherwise achieves only transient mixed hematopoietic chimerism and which has been extensively characterized ${ }^{8-10}$. We tested the hypothesis that the addition of expanded recipient Treg cells to the "standard" conditioning protocol would promote durable chimerism and allow acceptance of a donor kidney after a marked delay of 4 months, when donor kidneys are uniformly rejected by transient chimeras prepared with this protocol ${ }^{27}$.

\section{MATERIALS AND METHODS}

\section{Animals}

Male adult cynos (Charles River Primates, Wilmington, MA and Sanofi-Synthelabo, Bridgewater, NJ) were used. All procedures were approved by the IACUC of Columbia 
University and Massachusetts General Hospital (MGH). Both are AAALAC international accredited institutions.

\section{Cynomolgus MHC genotyping}

PBMCs were genotyped at the University of Wisconsin Primate Research Center Laboratory http://www.primate.wisc.edu/wprc/services/genetics.html ${ }^{28-31}$.

\section{Conditioning regimen}

Recipients of MHC mismatched donor BMT (Table 1 and Supplemental Figure 1) underwent the "standard" conditioning regimen as previously described $6,32+/-$ Treg (Figure 1A). Cyclosporine levels were maintained between $200-400 \mathrm{ng} / \mathrm{mL}$.

\section{Treg sorting and expansion}

The 1.0\% of CD4+ T cells expressing the highest levels of CD25 were sorted (FACSAria or Influx, BD Biosciences, Billerica, MA) and plated $\left(1 \times 10^{5} \mathrm{cells} / \mathrm{cm}^{2}\right)$ on fibroblasts (L929) $\left(10 \times 10^{5}\right.$ cells $\left./ \mathrm{cm} 2\right)$ expressing human CD32 (FcR), CD58 (LFA-3) and CD80 ${ }^{33,34}$ (referred as artificial APCs (aAPC)) in combination with human recombinant IL-2 (200 U/ml), antiCD3 (SP-34) 100ng/mL and rapamycin $100 \mu \mathrm{g} / \mathrm{mL}$ (Sigma-Aldrich, St Louis, MO) for 7 days. Growth medium consisted of RPMl-1640 (Gibco), fetal calf serum (FCS) (Gibco), Lglutamine, penicillin/streptomycin and nonessential amino acids. After 7 days, cells were replated with irradiated donor PBMCs (1 PBMC to 1 Treg) and IL-2 (200 U/ml), anti-CD3 (SP-34) $1 \mu \mathrm{g} / \mathrm{ml}$ or alternatively in combination with aAPCs $\left(3 \times 10^{5}-5 \times 10^{5}\right.$ cells per $\left.\mathrm{cm}^{2}\right)$ (Table 2). Cells were cultured for another 7 days, then split and cultured for another 7 days. When irradiated aAPCs were used, Treg were cultured for an additional 5 days in the presence of rapamycin $100 \mathrm{ng} / \mathrm{ml}$ (Table 2). After expansion, cells were cryopreserved in FCS (Gibco) with 5\% DMSO for future use.

\section{BMT}

Bone marrow (BM) was harvested aseptically from donor iliac bones by multiple percutaneous aspirations or surgically from the vertebrae. BM cells $\left(1.3-3.0 \times 10^{8}\right.$ mononuclear cells $/ \mathrm{kg}$ ) were infused intravenously. $\mathrm{CD} 34^{+}$content was $1 \%(+/-0.4 \%)$, as determined by flow cytometry.

\section{Kidney transplantation}

The details of the kidney transplant procedure were reported previously ${ }^{35}$. Kidneys were transplanted between day 119-134 post BMT. Recipients underwent unilateral native nephrectomy and ligation of the contralateral ureter on the day of transplant. The remaining native kidney was removed $\sim 100$ days after transplantation.

\section{Flow cytometric analyses, detection of chimerism and cell sorting}

Whole blood was lysed and labeled with a combination of the following mAbs: CD3 PerCPCy5.5 (SP34.2), CD4-APC (L200), CD4-PE (L200), CD8-APC (SK1), CD11b-PE (ICRF44). CD20-PE (2H7), CD25-PE (BC96), CD31-PE (WM59), CD56-PE (MY31), panMHC A.B.C-PE (W6/32), FOXP3-PE (236A/E7). For chimerism analysis, we used H38 
(anti-BW6, One Lambda, Inc., Canoga Park, CA, USA). The recipient and donor pairs were chosen based on their MHC haplotypes and $\mathrm{H} 38$ expression. The fluorescence of the stained samples was analyzed using FACS Calibur and FlowJo software.

\section{Mixed lymphocyte reactions (MLRs) and Treg suppression assays}

MLRs were performed as previously described ${ }^{6}$. In addition, Treg were titrated for their specificity in suppressing host anti-donor versus third party and donor anti-host versus third party responses. Donor or host PBMC responders were stimulated with irradiated host, donor or third party PBMCs. Host nonirradiated Treg were added to the culture and pulsed with tritiated thymidine 4 days after initiation of culture and read in a beta counter as previously described (6). Treg were also tested for suppression of anti-CD2-, -CD3- and CD28-coated NHP activation bead-mediated activation (Miltenyi Biotec) at 1 bead to every 2 PBMCs.

\section{RESULTS}

\section{Expansion, phenotype and suppression of cyno CD4+CD25high cells}

An average of 118,906.88 $\pm 9,588$ CD4+CD25high cells were sorted from each blood draw. Usually a 10-100 fold expansion was achieved within the first $7\left(0.695 \times 10^{6} \pm 0.175 \times 10^{6}\right)$ to $14\left(22.47 \times 10^{6} \pm 4.3 \times 10^{6}\right)$ days of culture (Figure $1 \mathrm{~b}$, representative lines). At the end of culture, Treg were analyzed for phenotype (Figure 1c) and function (Figures 2a,b) before cryopreservation.

Infused Treg expressed high levels of FOXP3 and CD25 (Figure 1c) (supplemental Table 1). Inhibition of the proliferation of bead- (anti-CD2/CD3/CD28) stimulated autologous (cryopreserved pretransplant) PBMCs generally revealed $>95 \%$ suppression at a 1:1 ratio of PBMCs:Treg (Figure 2a and 2b). The infused Treg varied in suppressive potency but all achieved 50\% suppression at or above a 1:2 Treg:PBMC ratio (Figure 2a and $\mathrm{b}$ ) (Supplemental Table 1).

We aimed to generate polyclonal, nonspecifically suppressive Treg lines with our expansion protocol. While donor PBMCs were added during the expansion period as a source of APCs, specificity studies on 2 different Treg lines (Figure 2c-f) revealed similar suppression of host anti-donor, host anti-third party, anti-host and donor anti-third party responses.

\section{Proof of concept that Treg infusion can prolong multilineage donor cell}

chimerism-We tested whether polyclonal Treg could prolong donor hematopoietic chimerism compared to controls, which historically achieved transient (30-60 days) chimerism ${ }^{6}$. Three control animals were treated as previously described ${ }^{6}$, except they did not receive a donor kidney graft on Day 0. Five animals received the same treatment plus Treg infusions posttransplant. These 5 animals (M5210, 90-39, 6c64, 6c1, 90-15) received expanded polyclonal autologous Treg $\left(15-53 \times 10^{6}\right.$ per infusion) during the first week posttransplant (days $0,2,5,7)$ and on day+50 (Supplemental Table 1). Total dose was $88-96 \times 10^{6} / \mathrm{kg}$. Two Treg recipients, M5210 and 90-39, developed significant multilineage chimerism (Figure 3 top row). While M5210 survived long-term, animal 90-39 died of CMV disease on day 43 with significant donor chimerism in all lineages (Figure 3). The 
chimerism in M5210 (Figure 3, top left panel) persisted longer than ever observed in this model, remaining detectable in the lymphoid, monocyte and granulocyte lineages until days 292, 224 and 335, respectively.

We monitored CMV viremia, and when it exceeded 10,000 copies/mL (initially) or 1,000 copies/mL (after our experience in the first few animals) we treated animals with Ganciclovir and/or Foscarnet. Animal 6c64 was given antiviral prophylaxis to prevent CMV reactivation and developed only low and short-lived chimerism (Figure 3) with prolonged pancytopenia, suggesting that BM-toxic effects of the antiviral treatment may have impaired both donor and recipient hematopoiesis. Two additional Treg recipients, $6 \mathrm{c} 1$ and 90-15, experienced CMV reactivation with high viral loads and required treatment with antivirals at high doses within the first week posttransplant These animals developed only short-lived and low levels of chimerism (6c64, 6c1, 90-15 shown in Figure 3, middle row) in association with protracted cytopenias, often requiring transfusions. These results suggest that CMV reactivation and/or the bone marrow toxic effects by early antiviral therapy may have potentially interfered with initial engraftment of the donor marrow.

Of the 3 control animals (90-47,90-7,90-1) receiving BMT without Treg infusion, 1 (animal 90-47) died of CMV prior to the development and implementation of the CMV surveillance and treatment protocol, without showing any significant chimerism (Figure 3 bottom row, left panel). The 2 other controls survived long-term. Animal 90-7 also developed low-level, short-lived chimerism. The third control animal, 90-1, was unique in that both it and the donor were negative for CMV (no additional CMV negative donor:recipient pairs were ever identified). Animal 90-1 (Figure 4 bottom row) showed multilineage chimerism that extended beyond the other controls but was within similar range as that previously reported in this model ${ }^{6,32}$.

In summary, recipients that reactivated CMV, regardless of Treg infusion, succumbed to disease if not treated promptly with antivirals. Early CMV reactivation and its treatment or prophylaxis were associated with very short-lived chimerism. Only 2 animals survived without antiviral treatment (1 Treg recipient and $1 \mathrm{CMV}$-negative non-Treg control.) The Treg recipient, M5210, had only a very low level CMV viremia ( $<1000$ copies $/ \mathrm{mL})$ and exhibited the longest documented donor chimerism ever seen with this or related protocols over a period of more than twenty years.

Only Treg recipients developed T cell chimerism-The 3 evaluable (ie that were not treated early with anti-virals) animals that had measurable lymphoid chimerism included Treg recipients M5210 and 90-39 and CMV(-) control recipient 90-1 (Figure 4). However, in the non-Treg recipient 90-1 (the control animal in which donor and recipient were CMV negative), lymphoid chimerism included NK cells (data not shown) and B cells (eg Figure $4 \mathrm{~b}$ left), but did not include significant donor $\mathrm{T}$ cell chimerism. In contrast, both evaluable Treg recipients had not only B cell and NK cell chimerism, but also significant CD4 and CD8 $\mathrm{T}$ cell chimerism (Figure 4b center and 4c right). In M5210, the long-lived Treg recipient, $\mathrm{T}$ cell chimerism first appeared 45 days post-BMT ( 2.5 weeks after cyclosporine had been discontinued) and increased significantly on day +60 post-BMT. Similarly, Treg recipient 90-39 (which died of CMV on day+43) had a spike in donor T cell chimerism in 
the peripheral blood 1 month after BMT (at the time immunosuppression was discontinued) (Figure $4 \mathrm{~b}$ right) and still had peripheral blood $\mathrm{T}$ cell chimerism (5\%, mostly in CD4 T cells) on the day of euthanasia. These results represent the first time that $\mathrm{T}$ cell chimerism has been observed using this nonmyeloablative monkey BMT model and suggest that Treg promote $\mathrm{T}$ cell chimerism.

We then investigated, in the only long-lived Treg recipient chimera, the phenotype of host and donor T cells, including CD31, a marker expressed on new thymic emigrants ${ }^{36}$ and CD45RA, a marker of naïve T cells, among both donor and recipient $\mathrm{T}$ cells (Figure 5). Almost all donor CD4 and CD8 T cells in M5210 expressed CD31throughout follow up (Figures 5a and 5b. Consistent with de novo origin in the recipient thymus, the expression of CD45RA was also very high on donor CD4+ T cells (Figure 5c), peaking close to $90 \%$. For recipient $\mathrm{T}$ cells, expression of $\mathrm{CD} 31$ in both $\mathrm{CD} 4$ and $\mathrm{CD} 8$ cell populations was significantly less. However, CD31 expression increased markedly in host T cells (80\%) early after the transplant (Figure $5 \mathrm{a}-\mathrm{b}$ ), suggesting that a wave of new host $\mathrm{T}$ cells was released from the thymus after transplant. The percentage of host-derived CD31+ T cells slowly decreased from day $+/-20$ until day +50 . CD45RA expression on recipient CD4+ Tcells peaked at 50\% at about 1 month posttransplant. The expression of CD31 and CD45RA was lower in animals that did not develop donor $\mathrm{T}$ cell chimerism, as shown in Figure 5 a-c (bottom rows) for animal 90-7, a control BMT recipient that did not receive Treg. In summary, donor T cells exhibited high levels of CD45RA and CD31, suggesting de novo development from the thymus in animal M5210.

\section{Kinetics of $C D 3+C D 4+F O X P 3+$ cells in the circulation following infusion-}

Peripheral Treg counts and percentages were similar in the 3 controls and the 2 evaluable Treg recipients that developed high levels of chimerism (M5210 and 90-39) (Figure 5d). Treg were largely of recipient origin (Supplemental Figure 2). A peak was observed on day +50 in M5210 after the infusion of 28 million Treg/ $\mathrm{kg}$, which was given in an effort to reverse a sudden increase in the absolute number of CD95+CD28- effector CD8 T cells (Figure $5 \mathrm{e}$ ) of mostly recipient origin (Figure $4 \mathrm{~b}$ middle panel) twenty days after the discontinuation of cyclosporine A (levels on day +48 were subtherapeutic at $118 \mathrm{ng} / \mathrm{mL}$ and low on day +53 at $35 \mathrm{ng} / \mathrm{mL}$ ). CD8 T cell counts declined after the infusion of Treg on day +50 (Figure 5e). Both the T cell chimerism (Figure 4b) and the myeloid chimerism (Figure 3 top left panel) increased shortly after the Treg infusion and remained stable for an additional 80 days (Figure 3 top left). Of note, on day 80 post BMT, there was a second increase in the absolute CD8 T cell count that was followed by a subsequent spontaneous Treg increase, after which CD8 counts normalized (Figure 5e).

In summary, infusion of Treg was associated with the development of $\mathrm{T}$ cell chimerism, prolonged multilineage chimerism and reversal of increasing recipient effector CD8+ T cell counts in animal M5210.

\section{Proof of principle; Persistent chimerism induced by Treg treatment was associated with in vitro donor-specific hyporesponsiveness and allograft} tolerance-M5210 showed donor-specific unresponsiveness in MLR at day 106, before kidney transplantation was performed (Figure 6b), whereas strong proliferative responses to 
the donor were present pre-BMT (Figure 6a). Similar responses to third party were observed pre and posttransplantation.

Animals were challenged with a solid organ allograft (a kidney from the same BMT donor) 4 months after the original BMT, without immunosuppression. Only 1 Treg recipient (M5210) was evaluable at the 4-month time point. At the time of kidney transplant, M5210 remained chimeric in all lineages (Figures 3 and 4). The recipient's contralateral ureter was ligated on day 0 and on day 100 postkidney transplant the recipient's contralateral kidney was removed. Serum creatinine levels (Figure 6e) remained normal and stable until the day of euthanasia 293 days postkidney transplant, demonstrating tolerance to the donor kidney. Histopathology on day+294 postkidney transplant showed no evidence for rejection in M5210 (Figure 6f).

Two control animals that underwent the same protocol (without Treg) were also grafted with a kidney from their same BM donor 4 months post-BMT. In contrast to M5210, the 2 controls rejected their donor kidneys within a month (Figure 6e), in line with previous results ${ }^{27}$. The donor kidneys in nonchimeric control animals showed Banff grade 3 rejection (Figure $6 \mathrm{f} \& \mathrm{~g}$ ) at the time of euthanasia. Nonchimeric Treg recipients (i.e. animals that received Treg but had short-lived hematopoietic chimerism in association with early CMV reactivation and treatment) retained anti-donor proliferative responses (Figure $6 \mathrm{c} \& d$ ) and rejected donor kidneys on day+120 post BMT $(n=2)$, similar to controls (data not shown). These results are proof of concept and suggestive of the importance of mixed chimerism in tolerance induction in this model. In contrast, no or minimal anti-donor alloantibody was detected in animals that rejected their donor kidneys (Supplemental Figure 3).

In summary, control recipients rejected the donor kidneys uniformly, while the only evaluable long-term surviving Treg recipient M5210 maintained normal kidney function until termination of the experiment. This result provides proof-of-principle that prolongation of chimerism using expanded Treg can promote more robust tolerance than that achieved in previous studies using this model ${ }^{6,27,32}$.

\section{DISCUSSION}

Our studies provide proof-of-concept that expanded recipient-derived polyclonal Treg can increase and extend donor hematopoietic chimerism and promote robust allograft tolerance across MHC barriers in a NHP nonmyeloablative BMT model without an increased risk of GVHD. Infusion of Treg is an attractive approach to overcoming HvG responses, as it may further reduce the risk of GVHD ${ }^{40}$ rather than increasing this risk or the overall toxicity of the conditioning regimen like most other approaches.

Phase I clinical trials using Treg have shown safety ${ }^{38}$, but efficacy remains to be proven. Both induced and natural Treg promoted engraftment, stable mixed chimerism and tolerance in mice under a minimal conditioning protocol in which the BM is otherwise rejected ${ }^{24,26}$. We demonstrate in a monkey model that host Treg improved the level and duration of chimerism, extending it to the T cells. Moreover, robust donor-specific tolerance was achieved in 1 evaluable animal such that a donor kidney grafted at 4-months posttransplant 
was accepted without immunosuppression. Previous studies using this protocol without Treg, donor BM and kidney co-transplanted on day 0 , were associated with long-term kidney graft survival in about $60 \%$ of animals ${ }^{6,39}$ and donor hematopoietic chimerism (in some animals reaching $+/-85 \%$ ) consistently disappearing by day 60 post $\mathrm{BMT}^{32}$. A delay in grafting a donor kidney to more than 3 months post-BMT was always associated with rejection of the donor graft 27 .

Previous work in NHPs (rhesus macaques) using a nonmyeloablative BMT regimen with costimulatory blockade achieved prolonged levels of donor chimerism as long as basiliximab and belatacept were infused. Chimerism was lost after discontinuation of this treatment ${ }^{41}$ and allograft tolerance was not achieved. In contrast, an animal in our study retained chimerism to 335 days and accepted a donor kidney grafted at 4 months, despite stopping immunosuppressive monotherapy at 28 days post-BMT.

In mice we have shown that the presence of $\mathrm{T}$ cell chimerism is associated with early and long-term deletional tolerance, because thymic engraftment of donor $\mathrm{T}$ cell progenitors reflects successful ablation of intrathymic alloreactivity, permitting intrathymic engraftment of both thymocyte progenitors and donor APCs that contribute to negative selection of donor-reactive $\mathrm{T}$ cells ${ }^{22,42-44}$. For the first time in the more than twenty years using this monkey nonmyeloablative BMT regimen, we have obtained evidence of de novo donor thymopoiesis, with $\mathrm{T}$ cell chimerism consisting of recent thymic emigrants in the peripheral blood. CD45RA and CD31 expression suggested that almost all donor cells were newlydeveloped, while recipient $\mathrm{T}$ cells were a mix of new thymic emigrants and naïve or memory $\mathrm{T}$ cells that evaded host conditioning.

CMV reactivation presented a major impediment to achieving the goals of our studies. While this complication has not been described in previous studies using the model we adopted, uniform CMV reactivation has been reported in cynomolgus monkeys receiving Thymoglobulin ${ }^{46}$. CMV reactivation was not directly caused by the infusion of Treg since control animals had a similar rate of reactivation. Only 1 animal, M5210, was able to control CMV without antiviral treatment. Because CMV itself can directly affect BM function ${ }^{47,48}$ and antiviral treatments are known to be BM toxic, modifications to the protocol are needed $^{49}$. The increased duration and level of chimerism observed in the $1 \mathrm{CMV}$-negative (non-Treg recipient) transplant (albeit without $\mathrm{T}$ cell chimerism or tolerance) supports a direct role for CMV and/or its treatment in limiting hematopoietic engraftment. We are currently exploring substitution of the mTOR inhibitor rapamycin for CSA in order to better control CMV reactivation and enhance Treg function, expansion and survival ${ }^{24,50}$.

In summary, we provide proof-of-concept that BM plus expanded cryopreserved polyclonal recipient Treg can prolong donor chimerism, promote $\mathrm{T}$ cell chimerism and induce robust tolerance without an increase of toxic conditioning intensity or GVHD risk in a preclinical monkey model. Successful refinement of this protocol has the potential to be translated to the clinic. 


\section{Supplementary Material}

Refer to Web version on PubMed Central for supplementary material.

\section{Acknowledgments}

We thank Dr. Remi Creusot for the critical review of the manuscript.

Funding for these studies was provided by NIH grant RO1OD017949, by startup funds from Columbia University Departments of Medicine and Surgery (to MS and RDS), the Banting Foundation (to MS), the Columbia University core award (to RDS), and the Irving Pilot Translational science award for new investigators (to RDS). Research reported in this publication was performed in the CCTI Flow Cytometry Core, supported in part by the Office of the Director, National Institutes of Health under awards S10RR027050. The content is solely the responsibility of the authors and does not necessarily represent the official views of the National Institutes of Health.

\section{ACRONYMS}

$\begin{array}{ll}\text { BMT } & \text { bone marrow transplant } \\ \text { CMV } & \text { cytomegalovirus } \\ \text { Cyno } & \text { cynomolgus macaque } \\ \text { HLA } & \text { human leukocyte antigen } \\ \text { MHC } & \text { major histocompatibility complex } \\ \text { MLR } & \text { mixed lymphocyte reaction } \\ \text { PBMCs } & \text { peripheral blood mononuclear cells } \\ \text { Treg } & \text { regulatory T cell }\end{array}$

\section{REFERENCES}

1. Hori S, Nomura T, Sakaguchi S. Control of regulatory T cell development by the transcription factor foxp3. Science. 2003; 299(5609):1057-1061. [PubMed: 12522256]

2. Kang SM, Tang Q, Bluestone JA. CD4+CD25+ regulatory T cells in transplantation: progress, challenges and prospects. Am J Transplant. 2007; 7(6):1457-1463. [PubMed: 17511675]

3. Sakaguchi S, Vignali DA, Rudensky AY, Niec RE, Waldmann H. The plasticity and stability of regulatory T cells. Nat Rev Immunol. 2013; 13(6):461-467. [PubMed: 23681097]

4. Krummey SM, Ford ML. Braking bad: novel mechanisms of CTLA-4 inhibition of T cell responses. American journal of transplantation : official journal of the American Society of Transplantation and the American Society of Transplant Surgeons. 2014; 14(12):2685-2690.

5. Amarnath S, Mangus CW, Wang JC, et al. The PDL1-PD1 axis converts human TH1 cells into regulatory T cells. Science translational medicine. 2011; 3(111):111ra120.

6. Kawai T, Cosimi AB, COLVIN RB, et al. Mixed allogeneic chimerism and renal allograft tolerance in cynomologous monkeys. Transplantation. 1995; 59:256-262. [PubMed: 7839449]

7. Kawai T, Cosimi AB, Spitzer TR, et al. HLA-mismatched renal transplantation without maintenance immunosuppression. New England Journal of Medicine. 2008; 358(4):353-361. [PubMed: 18216355]

8. Kawai T, Sogawa H, Koulmanda M, et al. Long-term islet allograft function in the absence of chronic immunosuppression: a case report of a nonhuman primate previously made tolerant to a renal allograft from the same donor. Transplantation. 2001; 72(2):351-354. [PubMed: 11477369] 
9. Kawai T, Cosimi AB, Wee SL, et al. Effect of mixed hematopoietic chimerism on cardiac allograft survival in cynomolgus monkeys. Transplantation. 2002; 73(11):1757-1764. [PubMed: 12084998]

10. Aoyama A, Ng CY, Millington TM, et al. Comparison of lung and kidney allografts in induction of tolerance by a mixed-chimerism approach in cynomolgus monkeys. TransplantProc. 2009; 41(1): 429-430.

11. Scandling JD, Busque S, Dejbakhsh-Jones S, et al. Tolerance and chimerism after renal and hematopoietic-cell transplantation. N Engl J Med. 2008; 358(4):362-368. [PubMed: 18216356]

12. Scandling JD, Busque S, Dejbakhsh-Jones S, et al. Tolerance and withdrawal of immunosuppressive drugs in patients given kidney and hematopoietic cell transplants. Am J Transplant. 2012; 12(5):1133-1145. [PubMed: 22405058]

13. Scandling JD, Busque S, Shizuru JA, Engleman EG, Strober S. Induced immune tolerance for kidney transplantation. NEnglJMed. 2011; 365(14):1359-1360.

14. Millan MT, Shizuru JA, Hoffmann P, et al. Mixed chimerism and immunosuppressive drug withdrawal after HLA- mismatched kidney and hematopoietic progenitor transplantation. Transplantation. 2002; 73(9):1386-1391. [PubMed: 12023614]

15. Leventhal J, Abecassis M, Miller J, et al. Chimerism and Tolerance Without GVHD or Engraftment Syndrome in HLA-Mismatched Combined Kidney and Hematopoietic Stem Cell Transplantation. SciTranslMed. 2012; 4(124):124ra128.

16. Leventhal J, Abecassis M, Miller J, et al. Tolerance induction in HLA disparate living donor kidney transplantation by donor stem cell infusion: durable chimerism predicts outcome. Transplantation. 2013; 95(1):169-176. [PubMed: 23222893]

17. Leventhal JR, Elliott MJ, Yolcu ES, et al. Immune reconstitution/immunocompetence in recipients of kidney plus hematopoietic stem/facilitating cell transplants. Transplantation. 2015; 99(2):288298. [PubMed: 25594553]

18. Ruedi E, Sykes M, Ildstad ST, et al. Antiviral T cell competence and restriction specificty of mixed allogeneic (P1+P2-->P1) irradiation chimeras. CellImmuol. 1989; 121:185-195.

19. Ildstad ST, Wren SM, Bluestone JA, Barbieri SA, Sachs DH. Characterization of mixed allogeneic chimeras. Immunocompetence, in vitro reactivity, and genetic specificity of tolerance. JExpMed. $1985 ; 162: 231-244$.

20. Zinkernagel RM, Althage A, Callahan G, Welsh RM Jr. On the immunocompetence of H-2 incompatible irradiation bone marrow chimeras. JImmunol. 1980; 124:2356-2365. [PubMed: 6444970]

21. Sharabi Y, Sachs DH. Mixed chimerism and permanent specific transplantation tolerance induced by a non-lethal preparative regimen. JExpMed. 1989; 169:493-502.

22. Tomita Y, Khan A, Sykes M. Role of intrathymic clonal deletion and peripheral anergy in transplantation tolerance induced by bone marrow transplantion in mice conditioned with a nonmyeloablative regimen. JImmunol. 1994; 153:1087-1098. [PubMed: 8027542]

23. Pilat N, Wekerle T. Mechanistic and therapeutic role of regulatory T cells in tolerance through mixed chimerism. CurrOpinOrgan Transplant. 2010

24. Pilat N, Baranyi U, Klaus C, et al. Treg-Therapy Allows Mixed Chimerism and Transplantation Tolerance Without Cytoreductive Conditioning. Am J Transplant. 2010; 10(4):751-762. [PubMed: 20148810]

25. Pilat N, Farkas AM, Mahr B, et al. T-regulatory cell treatment prevents chronic rejection of heart allografts in a murine mixed chimerism model. The Journal of heart and lung transplantation : the official publication of the International Society for Heart Transplantation. 2014; 33(4):429-437.

26. Pilat N, Klaus C, Hock K, et al. Polyclonal Recipient nTregs Are Superior to Donor or Third-Party Tregs in the Induction of Transplantation Tolerance. J Immunol Res. 2015; 2015:562935. [PubMed: 26273682]

27. Kawai T, Poncelet A, Sachs DH, et al. Long-term outcome and alloantibody production in a nonmyeloablative regimen for induction of renal allograft tolerance. Transplantation. 1999; 68:1767-1775. [PubMed: 10609955]

28. Campbell KJ, Detmer AM, Karl JA, et al. Characterization of $47 \mathrm{MHC}$ class I sequences in Filipino cynomolgus macaques. Immunogenetics. 2009; 61(3):177-187. [PubMed: 19107381] 
29. O'Connor SL, Blasky AJ, Pendley CJ, et al. Comprehensive characterization of MHC class II haplotypes in Mauritian cynomolgus macaques. Immunogenetics. 2007; 59(6):449-462. [PubMed: 17384942]

30. Pendley CJ, Becker EA, Karl JA, et al. MHC class I characterization of Indonesian cynomolgus macaques. Immunogenetics. 2008; 60(7):339-351. [PubMed: 18504574]

31. Wiseman RW, Karl JA, Bimber BN, et al. Major histocompatibility complex genotyping with massively parallel pyrosequencing. Nat Med. 2009; 15(11):1322-1326. [PubMed: 19820716]

32. Kawai T, Sogawa H, Boskovic S, et al. CD154 blockade for induction of mixed chimerism and prolonged renal allograft survival in nonhuman primates. Am J Transplant. 2004; 4(9):1391-1398. [PubMed: 15307826]

33. de Waal Malefyt R, Verma S, Bejarano MT, Ranes-Goldberg M, Hill M, Spits H. CD2/LFA-3 or LFA-1/ICAM-1 but not CD28/B7 interactions can augment cytotoxicity by virus-specific CD8+ cytotoxic T lymphocytes. European journal of immunology. 1993; 23(2):418-424. [PubMed: 7679643]

34. Levings MK, Sangregorio R, Galbiati F, Squadrone S, de Waal MR, Roncarolo MG. IFN-alpha and IL-10 induce the differentiation of human type $1 \mathrm{~T}$ regulatory cells. JImmunol. 2001; 166(9): 5530-5539. [PubMed: 11313392]

35. Benedict Cosimi A, Delmonico FL, Wright JK, et al. Prolonged survival of nonhuman primate renal allograft recipients treated only with anti-CD4 monoclonal antibody. Surgery. 1990; 108:406-414. [PubMed: 2382234]

36. Kimmig S, Przybylski GK, Schmidt CA, et al. Two subsets of naive T helper cells with distinct T cell receptor excision circle content in human adult peripheral blood. JExpMed. 2002; 195(6):789794.

37. Li HW, Sykes M. Emerging concepts in haematopoietic cell transplantation. Nat Rev Immunol. 2012; 12(6):403-416. [PubMed: 22627859]

38. Tang Q, Bluestone JA. Regulatory T-cell therapy in transplantation: moving to the clinic. Cold Spring Harbor perspectives in medicine. 2013; 3(11)

39. Kimikawa M, Sachs DH, COLVIN RB, Bartholemew A, Kawai T, Cosimi AB. Modifications of the conditioning regimen for achieving mixed chimerism and donor-specific tolerance in cynomolgus monkeys. Transplantation. 1997; 64:709-716. [PubMed: 9311707]

40. Sawitzki B, Brunstein C, Meisel C, et al. Prevention of graft-versus-host disease by adoptive T regulatory therapy is associated with active repression of peripheral blood Toll-like receptor 5 mRNA expression. Biol Blood Marrow Transplant. 2014; 20(2):173-182. [PubMed: 24184334]

41. Kean LS, Adams AB, Strobert E, et al. Induction of chimerism in rhesus macaques through stem cell transplant and costimulation blockade-based immunosuppression. Am J Transplant. 2007; 7(2):320-335. [PubMed: 17241112]

42. Tomita Y, Khan A, Sykes M. Mechanism by which additional monoclonal antibody injections overcome the requirement for thymic irradiation to achieve mixed chimerism in mice receiving bone marrow transplantation after conditioning with anti-T cell mAbs and 3 Gy whole body irradiation. Transplantation. 1996; 61:477-485. [PubMed: 8610364]

43. Tomita Y, Sachs DH, Khan A, Sykes M. Additional mAb injections can replace thymic irradiation to allow induction of mixed chimerism and tolerance in mice receiving bone marrow transplantation after conditioning with anti-T cell mAbs and 3 Gy whole body irradiation. Transplantation. 1996; 61:469-477. [PubMed: 8610363]

44. Nikolic B, Khan A, Sykes M. Induction of tolerance by mixed chimerism with nonmyeloblative host conditioning: the importance of overcoming intrathymic alloresistance. BiolBlood Marrow Transplant. 2001; 7(3):144-153.

45. Nadazdin O, Boskovic S, Murakami T, et al. Host alloreactive memory T cells influence tolerance to kidney allografts in nonhuman primates. SciTranslMed. 2011; 3(86):86ra51.

46. Han D, Berman DM, Willman M, et al. Choice of immunosuppression influences cytomegalovirus DNAemia in cynomolgus monkey (Macaca fascicularis) islet allograft recipients. Cell Transplant. 2010; 19(12):1547-1561. [PubMed: 20587138] 
47. Steffens HP, Podlech J, Kurz S, Angele P, Dreis D, Reddehase MJ. Cytomegalovirus inhibits the engraftment of donor bone marrow cells by downregulation of hemopoietin gene expression in recipient stroma. J Virol. 1998; 72(6):5006-5015. [PubMed: 9573270]

48. Paulin T, Ringden O, Lonnqvist B. Faster immunological recovery after bone marrow transplantation in patients without cytomegalovirus infection. Transplantation. 1985; 39(4):377384. [PubMed: 2984810]

49. Fries BC, Khaira D, Pepe MS, Torok-Storb B. Declining lymphocyte counts following cytomegalovirus (CMV) infection are associated with fatal CMV disease in bone marrow transplant patients. Exp Hematol. 1993; 21(10):1387-1392. [PubMed: 8395404]

50. Battaglia M, Stabilini A, Roncarolo MG. Rapamycin selectively expands CD4+CD25+FoxP3+ regulatory T cells. Blood. 2005; 105(12):4743-4748. [PubMed: 15746082]

51. Page A, Srinivasan S, Singh K, et al. CD40 Blockade Combines with CTLA4Ig and Sirolimus to Produce Mixed Chimerism in an MHC-Defined Rhesus Macaque Transplant Model. Am J Transplant. 2011 
A

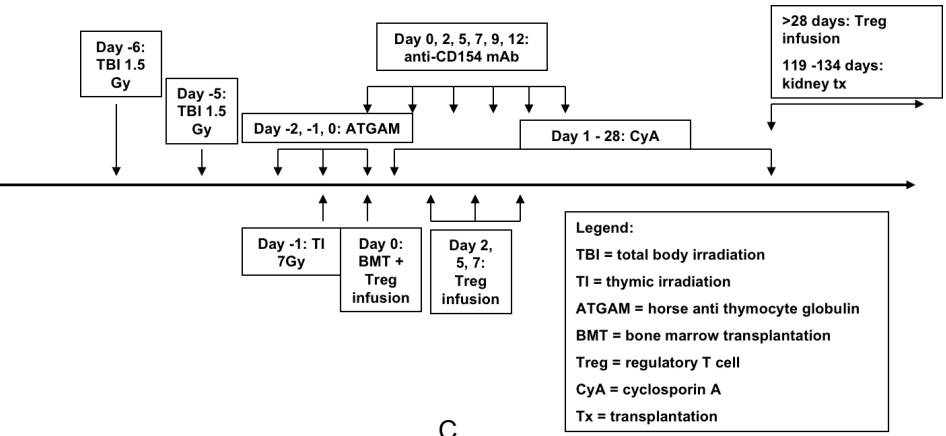

B

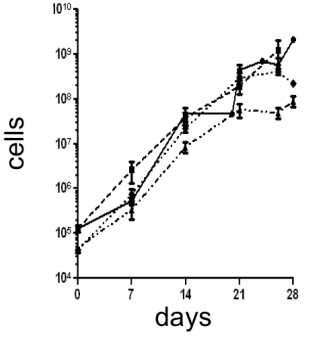

C CyA $=$ cyclosporin

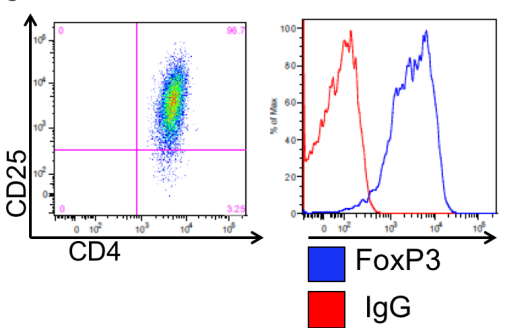

Figure 1.

Transplant scheme and Treg expansion. (A) Transplant protocol. (B). Expansion of Treg lines from 4 animals over 4 weeks. The average number of cells for each animal at each expansion time point is graphed (SEM) (bars). (C) A representative phenotype of Treg at the end of culture, with high levels of CD25 and of FOXP3. 
A
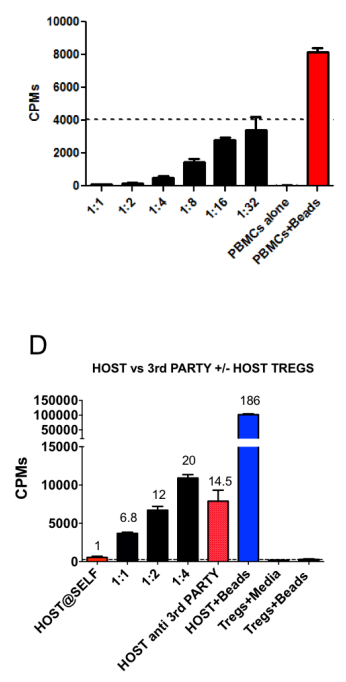

B

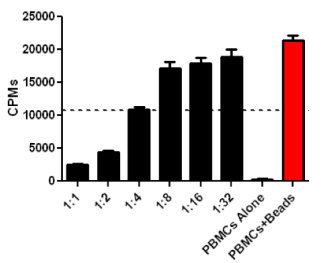

E

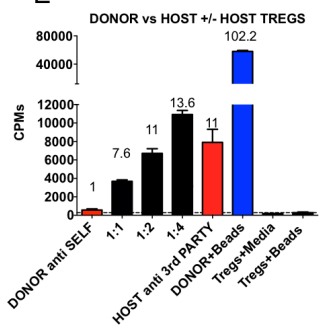

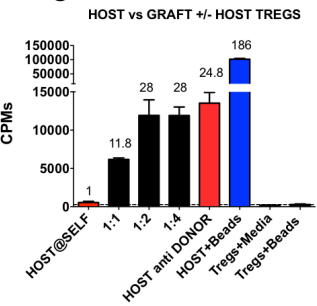

$\mathrm{F}$

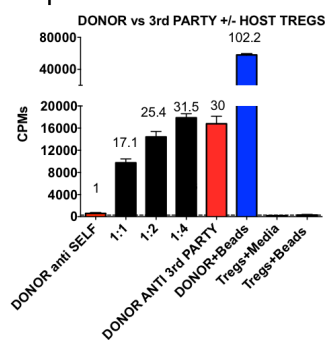

Figure 2.

Culture of cynomolgus regulatory T cells. (A) Highest quality Treg suppressed over $50 \%$ the proliferation of bead stimulated (anti-CD2CD3CD28) PBMCs at 1:32 Treg:PBMC ratio. (B) All Treg lines achieved at least 50\% suppression of proliferation at a 1:2 Treg:PBMC ratio. Microsuppression assays shown. (C-F) MLRs assessing the specificity of host Treg. Host (C,D) and donor (E,F) PBMC responders were plated with either host, donor or third party stimulators. Host Treg were added to the cultures at the indicated PBMC:Treg ratios (1:1, 1:2 and 1:4) and assessed for suppressive activity. All data points represent means of triplicates. Error bars indicate SE. Similar results were obtained in a repeat experiment (not shown). 


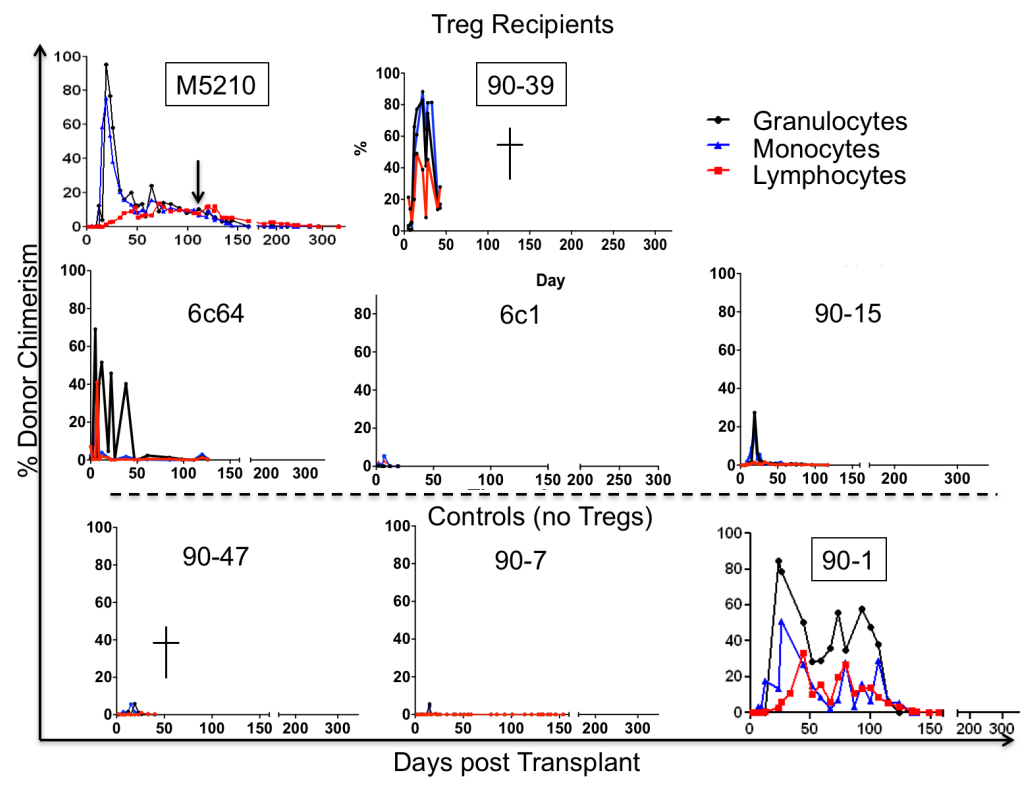

Figure 3.

Summary of percent donor chimerism. Granculocyte (black circles), monocyte (blue triangles) and lymphocyte (red squares) lineages of each animal are shown. Animals with boxed identification numbers (M5210, 90-39, and 90-1) received no antiviral treatments. Animals whose figures have a cross died or were euthanized due to untreated or unmanageable CMV disease. Animal 90-47 was serologically CMV- pre-Tx, but developed CMV following BMT from a CMV+ donor that had been serologically negative on initial screen. Animal 90-1, who was CMV-pre-Tx and received a BMT from a CMV-donor, never developed CMV. The 5 animals shown on the top part of the figure received BMT+Treg, while the 3 below the dotted line received BMT without Treg. 
A
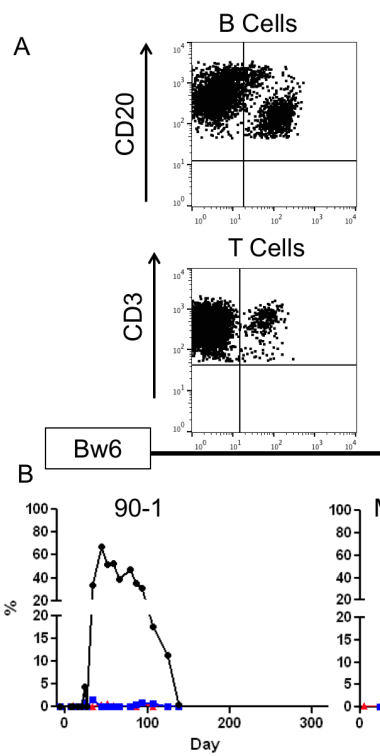

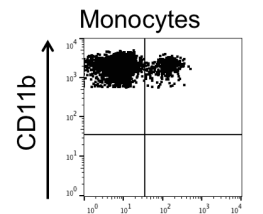

CD4+CD8- T Cells

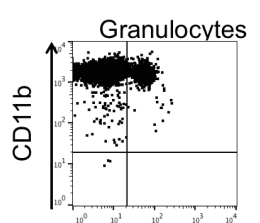

CD4-CD8+T Cells

Figure 4.

Chimerism analysis of BMT recipients. (A) Representative flow cytometry of animal M5210 on day+99 post BMT. Donor chimerism is measured with the Bw6+ (MHC-I) marker. B cell $(\mathrm{CD} 20+)$, monocyte (CD11b+) and T cell (CD3, CD4 and CD8) chimerism measured among cells with low/medium forward and side scatter (not shown). Granulocytes (CD11b+) were analyzed among cells with high forward and side scatter (not shown). (B) B Cell (black circle), CD4 (red triangle) and CD8 (blue square) T cell chimerism in the 3 animals with the highest and most prolonged chimerism. CMV- control animal 90-1 developed high levels B cell chimerism, but no $\mathrm{T}$ cell chimerism and chimerism declined after discontinuation of immunosuppression and was completely lost by day 110. Treg recipient M5210 developed high B cells chimerism and delayed, prolonged T cell chimerism in both CD4 and CD8 T cell lineages. Chimerism lasted over 300 days post BMT. Treg recipient 90-39 was euthanized at day 43 due to CMV disease. At the time of euthanasia B cell and CD4 T cell chimerism was detectable in the peripheral blood.

Transplantation. Author manuscript; available in PMC 2018 February 01. 


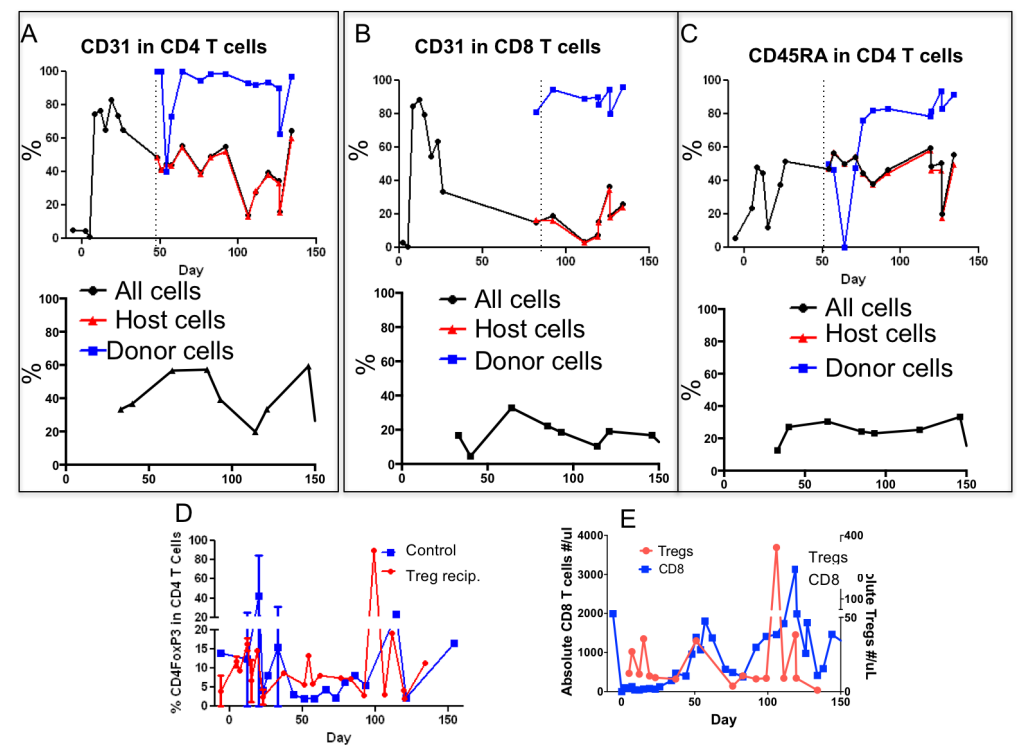

Figure 5.

Immune reconstitution of animal M5210 Post BMT (+Treg) and 90-7 (control). (A-C top row M5210 and bottom row 90-7 control). Dotted lines indicate first detection of donor chimerism in CD4 or CD8 T cells. (A-top) Total CD4 T cells (black circle) expressed CD31 at increased levels posttransplant. Donor CD4 T cells (blue squares) maintained high CD31expression. Recipient-derived CD4 T cells (red triangles) expressed lower levels of CD31. (B-top) Total CD8 T cells expressed high CD31 levels following transplant (black circle). CD31 expression in host CD8 T cells (red triangles) decreased, however donorderived cells (blue squares) maintained high CD31 expression long after transplant. (C-top) Donor-derived CD4 T cells (blue squares) expressed higher levels of CD45RA compared to recipient CD4 $\mathrm{T}$ cells (red triangles). In contrast animal 90-7 never exhibited any donor chimerism (A-C bottom row) and the levels of CD31 and CD45 were lower than those observed in M5210 donor (blue) T cell populations. (D) Average percentage of CD4 T cells expressing FOXP3 after transplant in Treg recipients (red triangles) ( $\mathrm{n}=2$ animals) compared to control animals (blue squares) $(n=3)$. (E) Animal M5210 absolute Treg numbers (red triangles) compared to the absolute number of CD8 T cells (blue squares). 
A

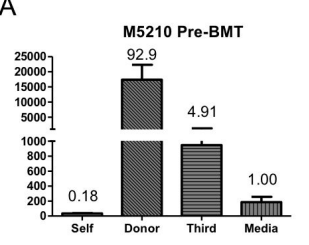

C

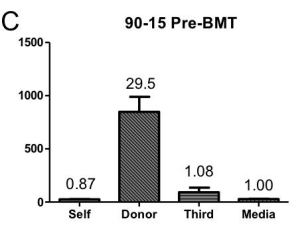

F

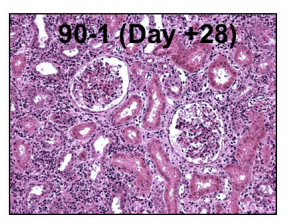

B
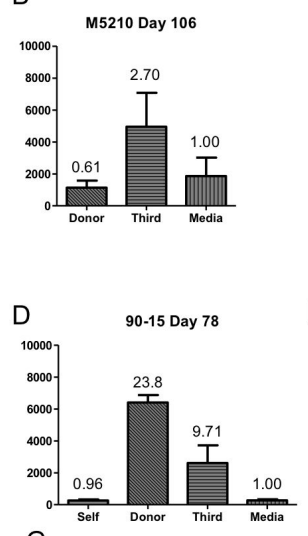

G

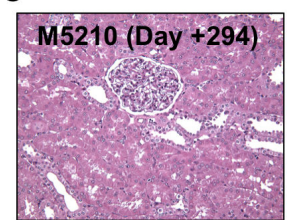

- Treg Recipient

- Control Animal 1

-4. Control Animal 2

E

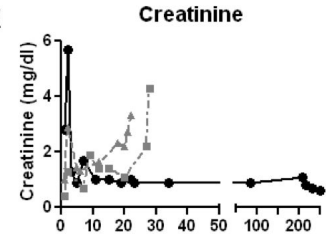

Day post kidney transplant

Figure 6.

Tolerance to the donor in M5210 Treg recipient but not in control. (A) Pre-transplant MLR in animal M5210 demonstrates strong proliferative responses to donor (diagonal stripes) and weaker response to third party (horizontal stripes). (B) Day 106 Post-Tx MLR (before kidney allograft from the same BM donor). Proliferation is maintained to third party but not to donor. (C) Animals that lost chimerism never became tolerant nor showed decreased antidonor proliferation. An example is shown in (C) pretransplant and (D) posttransplant day 78. (E) Following kidney transplant, creatinine levels in M5210 (black circle) stayed in the normal range while that in 2 control (non-Treg) animals (dashed grey lines) showed increases 2-3 weeks following kidney transplant. (F,G) Kidney histopathology. Biopsies were taken from transplanted donor kidneys at the time of euthanasia. Shown is day+28 and day+294 (day of euthanasia) post kidney transplant in animals 90-1 (F) (control, left) and M5210 (G) (+Treg, right) respectively. 90-1 had extensive lymphocyte infiltrates scored as a Banff grade 3 rejection, while M5210 showed no signs of rejection. 


\section{Table 1}

Donor: recipient MHC mismatches (refer to Supplemetal Figure 1). All recipients had donors with 1 or both MHC-I and II alleles mismatched.

\begin{tabular}{|l|l|c|l|l|}
\hline Recipient & Tregs & CMV (serology) & Mismatch in MHC-I & Mismatch in MHC-II \\
\hline $90-47$ & No & + & 2 haplotype & 1 haplotype \\
\hline $90-1$ & No & - & 2 haplotype & 1 haplotype \\
\hline $90-7$ & No & + & 2 haplotype & 1 haplotype \\
\hline M5210 & Yes & $+*$ & 2 haplotype & 1 haplotype \\
\hline $90-39$ & Yes & - & 1 haplotype & 2 haplotype \\
\hline $90-15$ & Yes & + & 1 haplotype & 1 haplotype \\
\hline $6 c 64$ & Yes & + & 1 haplotype & 2 haplotype \\
\hline $6 c 1$ & Yes & + & 1 haplotype & 1 haplotype \\
\hline
\end{tabular}

*Animal M5210 was not tested but assumed to be positive. 


\section{TABLE 2}

Treg protocols

\begin{tabular}{|c|c|c|c|c|}
\hline Reagents & Day 0 & Day 7 & Day 14 & Day 21 \\
\hline IL-2 & $\checkmark$ & $\checkmark$ & $\checkmark$ & \\
\cline { 1 - 4 } Anti-CD3 & $\checkmark$ & $\checkmark$ & $\checkmark$ & \\
\cline { 1 - 3 } Donor PBMCs & - & $\checkmark$ & $\checkmark$ & \multirow{2}{*}{ FREEZE } \\
\cline { 1 - 3 } Artificial APCs & $\checkmark$ & - & - & \\
\hline Rapamycin & $\checkmark$ & - & - & \\
\hline
\end{tabular}

$\frac{\text { 市 }}{\text { 을 }}$

\begin{tabular}{|c|c|c|c|c|c|}
\hline Reagents & Day 0 & Day 7 & Day 14 & Day 21 & Day 26 \\
\hline IL-2 & $\checkmark$ & $\checkmark$ & $\checkmark$ & $\checkmark$ & \\
\cline { 1 - 5 } Anti-CD3 & $\checkmark$ & $\checkmark$ & $\checkmark$ & $\checkmark$ & \multirow{2}{*}{ FREEZE } \\
\cline { 1 - 4 } Donor PBMCs & - & $\checkmark$ & $\checkmark$ & $\checkmark$ & \multirow{2}{*}{$\checkmark$} \\
\cline { 1 - 4 } Artificial APCs & $\checkmark$ & $\checkmark$ & $\checkmark$ & $\checkmark$ & \\
\hline Rapamycin & $\checkmark$ & - & - & $\checkmark$ & \\
\cline { 1 - 4 }
\end{tabular}

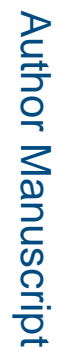

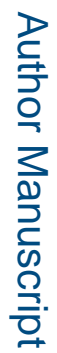

Transplantation. Author manuscript; available in PMC 2018 February 01. 